PEB Échanges, Programme pour la construction et l'équipement de l'éducation 2001/15

\title{
L'impact du temps sur la conception des environnements pédagogiques
}

\author{
Prakash Nair
}

https://dx.doi.org/10.1787/760257417627 


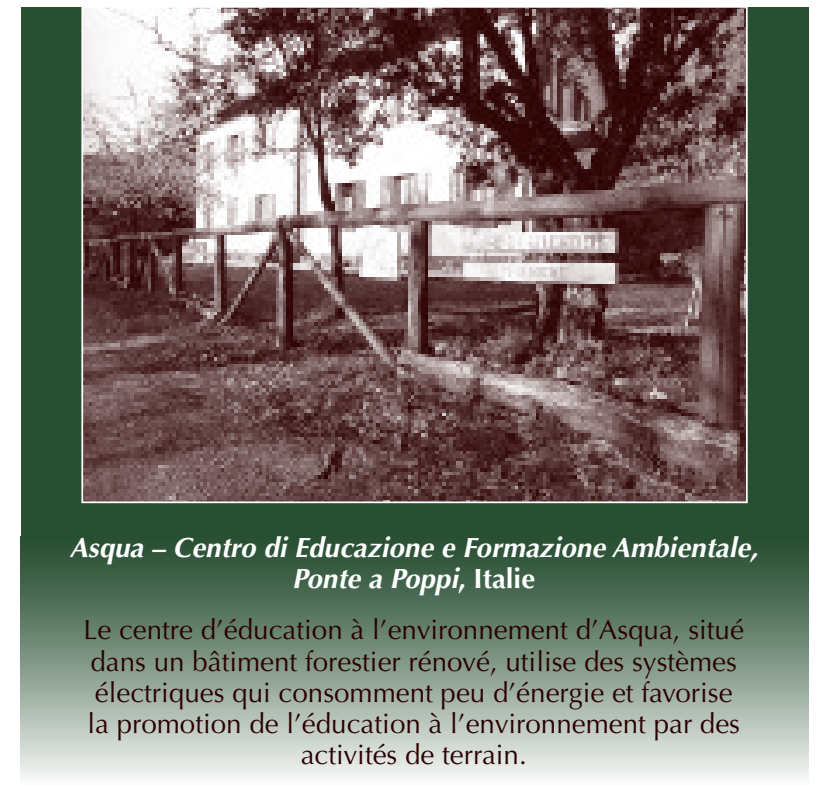

\section{Conclusion}

On peut faire ressortir, pour conclure, trois points principaux :

- Récapitulés sur plusieurs années, tout spécialement sur la décennie qui s'achève, les travaux du PEB (séminaires et publications) apparaissent comme autant de jalons, ou de pièces d'un puzzle, qui, ensemble, dessinent une perspective et qui expriment effectivement une vision de l'école d'aujourd'hui et de demain.

- Cette vision recoupe amplement - on I'avait indiqué en introduction - l'approche de « l'école intelligente » développée par les divers intervenants italiens à Milan, tout spécialement par les représentants du Centre pour l'innovation et l'expérimentation éducatives de Milan (CISEM) ; la convergence de vues, à cet égard, est manifeste.

- L'approche du PEB ne constitue nullement une vision " imposée »; elle se " construit » à partir des contributions des pays et organismes participant au Programme ; car le PEB est un carrefour, un forum de discussion et d'échanges au service de toutes les parties prenantes : États membres, collectivités territoriales, organismes d'études ; il s'agit bien de tirer parti des expériences et innovations des uns et des autres, ne serait-ce après tout que pour éviter la répétition d'erreurs éventuelles.

Les contributions financières pour ce Programme sont limitées parce que son budget est modeste; mais dans la réflexion sur l'école de demain, au regard des enjeux pour nos systèmes éducatifs, et plus largement nos sociétés, un tel programme occupe une place certainement utile.

\section{François Louis}

Président du comité de direction du PEB

Programme de l'OCDE pour la construction et

l'équipement de l'éducation

Télécopie : 33 (0)155551001

francois.louis@education.gouv.fr

\section{L'IMPACT DU TEMPS SUR LA CONCEPTION DES ENVIRONNEMENTS PÉDAGOGIQUES}

Cet article est un compte rendu de l'atelier de travail sur le Temps organisé dans le cadre de la conférence internationale "Des environnements pédagogiques novateurs " qui s'est tenue en novembre $2000^{\top}$. Ce compte rendu, rédigé par Prakash Nair, expert américain en installations et techniques scolaires, bénéficie fortement de la contribution de Hans F. Van Aalst, co-dirigeant de l'atelier de travail, et de tous les participants concernés.

\section{Introduction}

Le temps joue un rôle fondamental dans le modelage des systèmes éducatifs et des établissements scolaires. Pourtant, c'est un élément dont l'impact est rarement pris en compte dans la conception des écoles car imposer des frontières temporelles à l'apprentissage est une tradition séculaire rarement remise en cause, bien qu'une multitude d'éléments indique qu'elle empêche maints élèves d'apprendre efficacement.

\section{Le temps modèle les écoles et dicte leur organisation}

Aux États-Unis, les matières sont enseignées au cours de périodes de temps clairement définies de 45-55 minutes. Les enfants sont regroupés par âge, chaque groupe suivant l'école pendant une " année » scolaire par classe d'enseignement, année elle-même divisée en trimestres ou "périodes de notation ». Il y a une longue période de vacances en été, une plus courte en hiver et au moins une autre au printemps. L'école se divise elle-même en cinq parcours limités dans le temps et appelés la maternelle (pour les moins de cinq ans), le jardin d'enfants, l'école

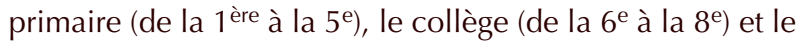
lycée (de la $9^{\mathrm{e}}$ à la $12^{\mathrm{e}}$ ). Ce système est celui en place aux États-Unis, mais il existe un système équivalent dans tout pays doté d'un système éducatif formel. Le programme qui détermine un modèle scolaire est orienté dans une grande mesure par ces contraintes temporelles prédéfinies.

\section{Des écoles sans rythme scolaire imposé}

Nous disposons maintenant de travaux de recherche montrant pourquoi l'idée selon laquelle il faut enseigner aux élèves la même chose au même moment et au même endroit n'est pas plausible. Il est de fait que

1. Voir PEB Échanges numéro 43, juin 2001, " Le tournant d'Amsterdam », pp. 12-17. 

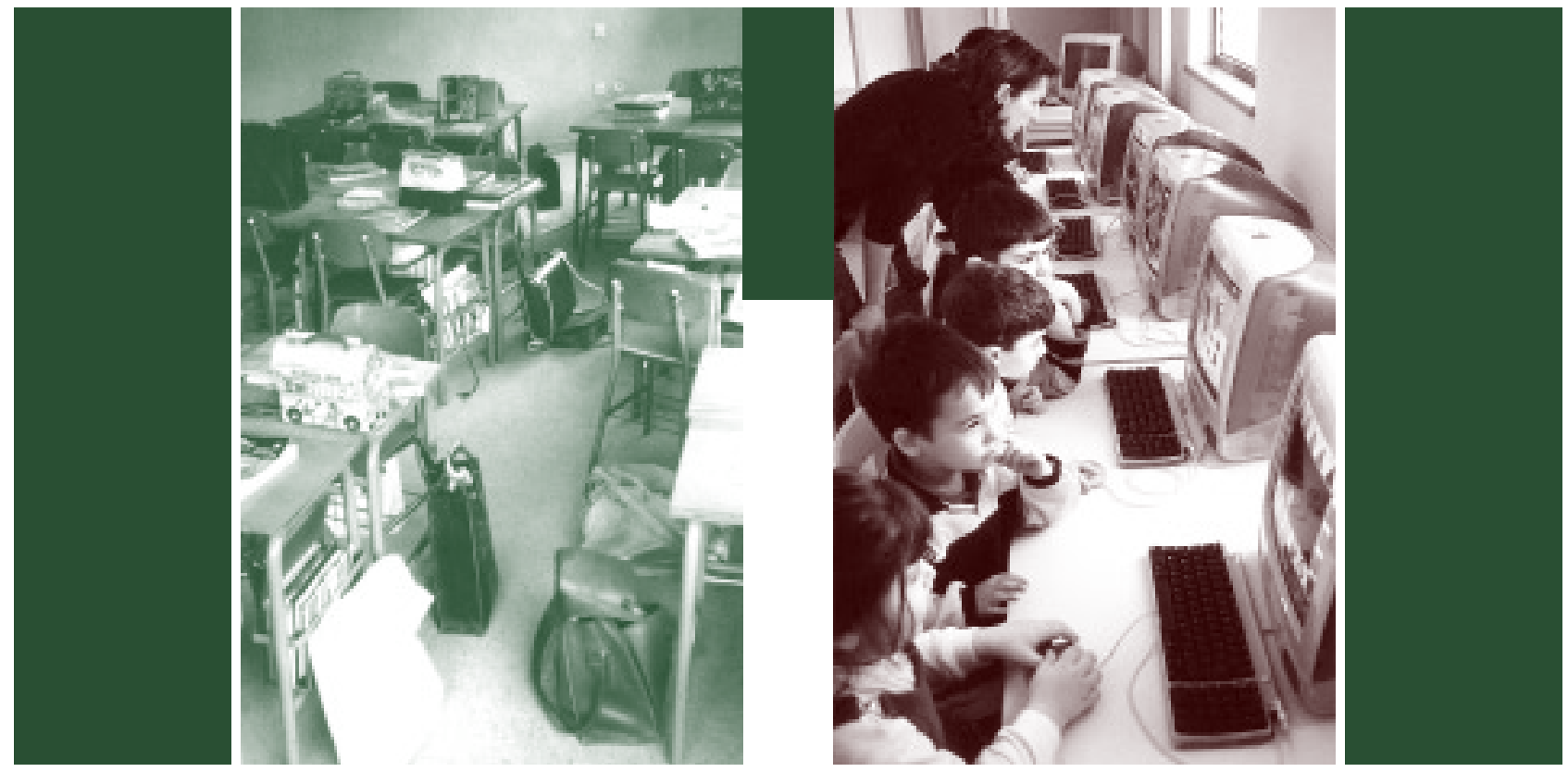

l'apprentissage ne commence ni ne s'arrête à l'école ; I'apprentissage à vie est une expression qui commence à entrer dans la conscience collective. Les locaux physiques où l'enseignement est dispensé doivent refléter la vision éducative actuelle empreinte de bon sens, à savoir qu'il faut beaucoup moins s'attacher au temps et beaucoup plus à révéler tout le potentiel de chaque élève au rythme de ce dernier.

\section{Vers l'école sans rythme scolaire imposé}

L'information ayant perdu le pouvoir qu'elle exerçait autrefois, l'objet de l'apprentissage est passé de la mémorisation à la réflexion et à l'analyse critiques, accompagnées d'un enseignement pratique fondé sur des projets. Même quand il est impossible d'apprendre par expérience dans un environnement réel, les simulations informatiques sont souvent utilisées pour reproduire les expériences de la vie réelle. La collaboration et le travail d'équipe et le fait que l'apprentissage déborde du cadre de l'école pour s'étendre à la collectivité extérieure sont autant de caractéristiques de l'école sans rythme scolaire imposé.

Il est important de se rappeler que le passage à des écoles sans rythme scolaire imposé fait entrer dans un paradigme très différent du paradigme à frontières temporelles que représente la majorité des locaux actuels dédiés à l'enseignement. Les éléments suivants sont quelques uns de ceux que les architectes et les autres parties prenantes devront prendre en compte lorsqu'ils bâtiront les écoles du XXI ${ }^{\mathrm{e}}$ siècle.

\section{Enseignement contre apprentissage}

Bien qu'utilisés souvent l'un pour l'autre, ces deux termes ne sont pas synonymes. Perçu traditionnellement comme un processus objectif, l'enseignement était réputé pouvoir être soigneusement segmenté en petites périodes de temps prédéterminées. L'hypothèse sous-jacente est que, à quelques exceptions près, la plupart des élèves apprendront à peu près les mêmes choses au cours des mêmes périodes de temps accordées. En d'autres termes, un enseignement homogène équivaut à un apprentissage homogène. Cette hypothèse est la pierre angulaire de la majorité des systèmes éducatifs et, par extension, les établissements scolaires représentent aussi cette approche temporelle segmentée de l'apprentissage.

\section{Horloge personnelle contre horloge sociétale}

Lorsque le paradigme à frontières temporelles de l'éducation a été créé, peu d'attention a été accordée à l'idée selon laquelle chaque être humain vit le temps de façon différente. En d'autres termes, le temps n'est pas objectif mais subjectif et expérimental. La même période de temps objective est perçue selon les individus comme étant plus longue ou plus courte selon leur degré d'intérêt et de concentration pour une quelconque activité donnée. Cela montre que l'apprentissage est quelque chose de très personnel, ce qui s'explique, entre autres, par le fait que chaque apprenant "interprète »les choses différemment sous l'influence des expériences qu'il ou elle a personnellement vécues au cours de sa vie.

Toute tentative visant à faire de l'apprentissage et du temps des constantes va à l'encontre de l'objectif de l'enseignement : donner à chaque élève une chance de réussir. Autrement dit, pour qu'un élève apprenne, il faut lui accorder le temps qui lui est nécessaire pour pleinement comprendre le sujet enseigné. Réciproquement, il est inévitable que certains élèves $\mathrm{n}^{\prime}$ apprennent pas lorsque le temps est maintenu constant. 


\section{L’apprentissage personnalisé}

L'idée selon laquelle chaque élève est unique et, par conséquent, que l'attention qui lui est accordée doit être adaptée à son cas, a donné naissance au mouvement en faveur de l'apprentissage personnalisé. Schématiquement, le concept d'apprentissage personnalisé reconnaît que l'éducation n'a de sens que dans le contexte des centres d'intérêt et capacités propres à chaque apprenant. Plutôt que de centrer les efforts sur l'identification des problèmes d'apprentissage et sur leur résolution (ce qui est le fondement des évaluations basées sur les tests), I'apprentissage personnalisé vise à révéler et maximiser le potentiel propre à chaque apprenant.

Pour que l'apprentissage personnalisé porte ses fruits, les élèves doivent être confrontés à des modes d'apprentissage différents. En effet, certains excelleront dans les modes où les capacités cognitives seront fortement mises à contribution tandis que d'autres excelleront dans les domaines où les capacités sociales et artistiques seront très sollicitées. Si réussir consiste à parvenir à réaliser son potentiel, alors, en toute logique, les systèmes éducatifs doivent être construits de manière à ce que l'apprentissage soit personnalisé.

En ce qui concerne les lieux d'enseignement, l'apprentissage personnalisé impliquera l'existence de domaines d'activité très différents non seulement dans la salle de cours mais aussi dans tout le reste de l'école. Naturellement, cette approche de l'apprentissage nécessitera une réforme radicale de l'administration temporelle de l'école - qu'il s'agisse des périodes de temps sur lesquelles l'organisation de la journée scolaire est basée ou du regroupement par âge des enfants, qui caractérisent le découpage des classes à l'école élémentaire, au collège et au lycée.

\section{Le temps en tant que variable dépendante}

Le problème des systèmes éducatifs à frontières temporelles est qu'ils considèrent le temps comme une variable indépendante. Tous les objectifs éducatifs déclarés sont de ce fait considérés par défaut comme étant des variables dépendantes subissant la tyrannie du temps.

La solution à ce problème consiste à faire du temps une variable dépendante. Autrement dit, les principaux objectifs éducatifs deviennent les variables indépendantes et le temps devient subordonné à la réalisation de ces objectifs.

Dans ce schéma, les variables suivantes sont quelques unes des variables indépendantes libérées des contraintes des frontières temporelles qui limitent leur réalisation en ce qui concerne les apprenants individuels. Cela ne signifie pas qu'il n'est pas enseigné aux élèves à gérer le temps, mais seulement que le temps ne peut être considéré comme

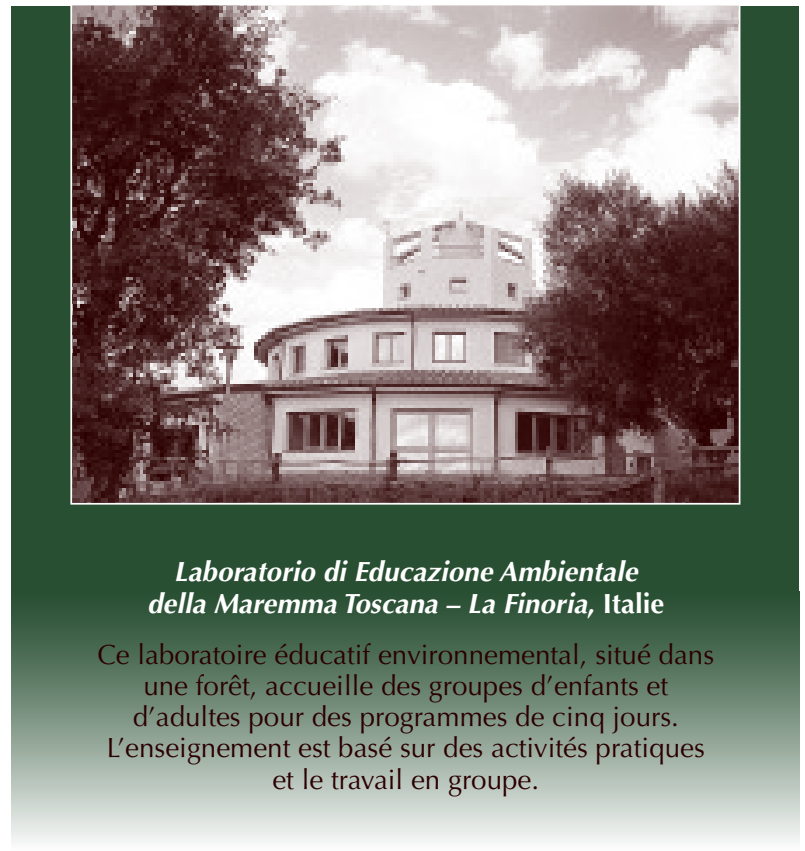

une variable indépendante des résultats escomptés. Ces variables indépendantes sont également autant d'exemples d'objectifs éducatifs importants pour le XXI ${ }^{\mathrm{e}}$ siècle :

- développer les capacités à gérer le temps ;

- entretenir la créativité ;

- encourager le travail de réflexion indépendant ;

- développer l'intelligence émotionnelle ;

- faire prendre conscience de soi ;

- entretenir des intelligences multiples ;

- acquérir des connaissances ;

- appliquer des connaissances ;

- créer des communautés ;

- inculquer des compétences utiles dans différentes disciplines.

\section{L’apprentissage basé sur un projet}

Dans ce schéma où le temps devient une variable subordonnée dans le processus d'apprentissage, I'apprentissage basé sur un projet peut remplacer l'apprentissage basé sur un sujet. Les projets sont un bon outil de gestion du temps car ce sont des unités organisationnelles qui permettent un développement holistique de la personne tout en étant le moyen de transmettre des compétences concrètes spécifiques. Grâce à l'apprentissage basé sur un projet, les élèves ne sont plus obligés d'absorber des informations hors contexte. Comme il a été montré, cet apprentissage par cœur ne procure aucun avantage à long terme. L'enseignement basé sur des projets concrets, en revanche, accroît le degré $d^{\prime}$ intérêt et de motivation des élèves et se traduit par un apprentissage dont I'influence perdure souvent. 


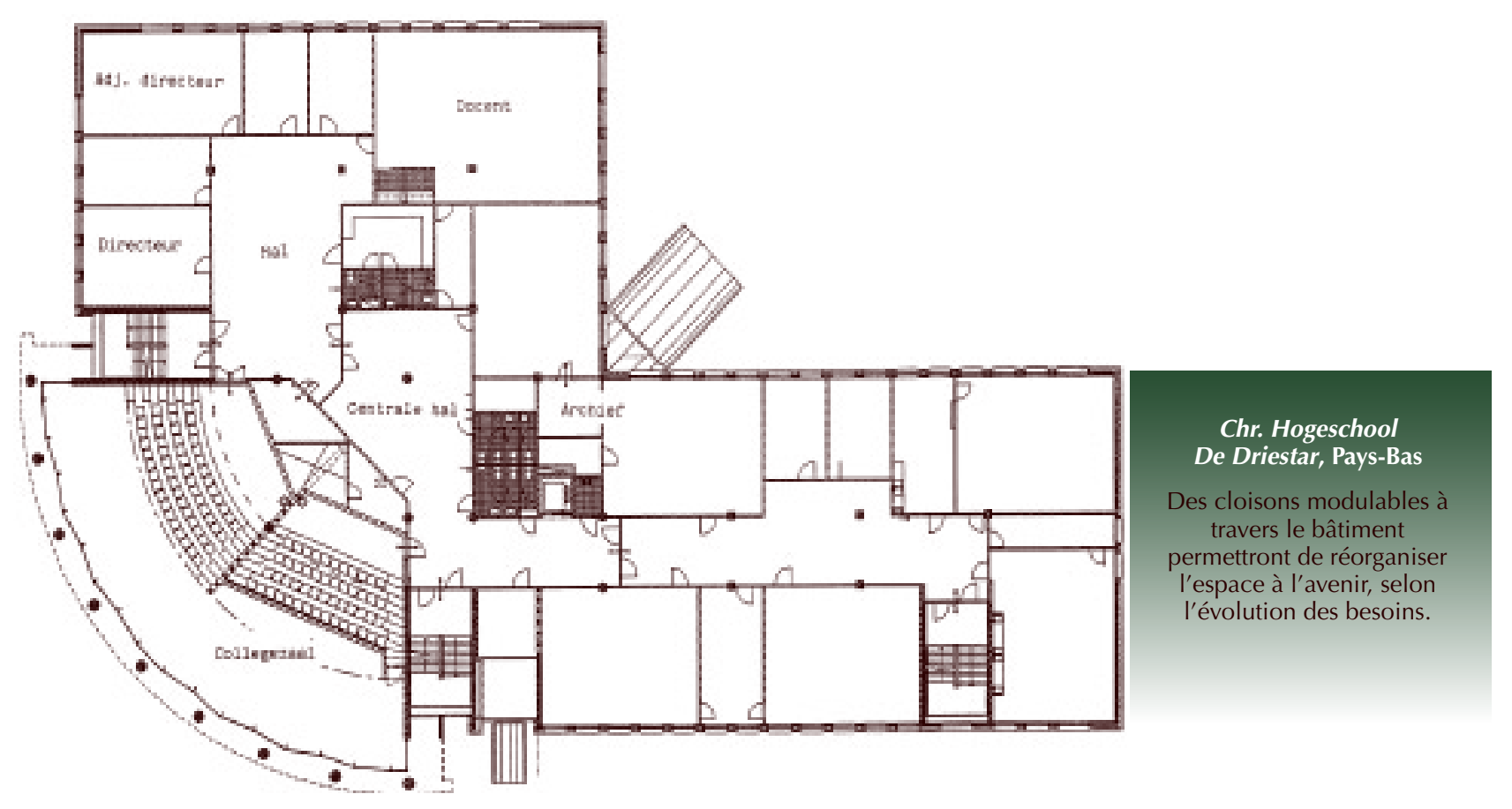

\section{L'apprentissage à vie peut s'effectuer à toute heure et en tout lieu}

Dissocier l'enseignement du strict respect d'éléments fondés sur le temps exige en outre de repenser le lieu de l'enseignement. Lorsqu'ils ne sont plus rythmés par la journée scolaire qui va de $9 \mathrm{~h}$ à $15 \mathrm{~h}$, les systèmes éducatifs doivent élargir le concept d'apprentissage afin qu'il couvre tant la notion de "à toute heure " que celle de " en tout lieu ». A partir du moment où les écoles offrent la possibilité de choisir entre des horaires et des cours différents, elles devront aussi inévitablement offrir la possibilité de choisir entre des lieux différents. Ceci est facilité par l'avènement de I'apprentissage virtuel sur Internet et l'utilisation répandue du courrier électronique, de la vidéoconférence et d'autres formes de communication électronique ne nécessitant pas que l'élève et l'enseignant soient physiquement présents au même endroit.

Dans le paradigme de l'apprentissage sans rythme scolaire imposé, l'établissement scolaire central, jusqu'ici le pilier de nos systèmes éducatifs, sera relégué au rang de lieu de rencontres physiques, mais ne sera pas nécessairement le lieu de tous les apprentissages.

\section{Rôle du mentor/coach dans l'école sans rythme scolaire imposé}

Dans les premières années de l'apprentissage, les enseignants aideront les apprenants à comprendre que, même lorsque le temps est flexible, il existe des frontières et structures auto-imposées. Savoir gérer le temps est une compétence qui doit être inculquée tôt dans la vie afin qu'il devienne naturel de l'exercer ultérieurement.
Les enseignants, en tant que conseillers et facilitateurs, s'efforcent de s'inscrire hors de l'équation de l'apprentissage car ils enseignent aux élèves l'art de l'indépendance et de l'auto-responsabilité.

$L^{\prime}$ « enseignant » n'est pas nécessairement un instructeur désigné mais peut être simplement toute personne capable de transmettre des connaissances. Dans les salles de cours et autres lieux d'apprentissage, l'enseignant peut souvent être un pair ou un autre mentor.

\section{D'un « temps figé » au " théâtre d'improvisation »}

Il est facile de comprendre pourquoi une école bâtie au début du siècle dernier apparaît aujourd'hui comme " figée dans le temps ", représentant un système éducatif inadapté à l'ère de l'information actuelle. II est plus difficile d'expliquer pourquoi les écoles construites dans les années 90 sont devenues si rapidement dépassées.

Dans la communauté des affaires, il est admis que la forme et le contenu du lieu de travail soient modifiés de fond en comble au bout de quelques années. Les locaux, par conséquent, sont de simples "coquilles» auxquelles ont été intégrés quelques éléments d'infrastructure de base comme les ascenseurs, I'alimentation en énergie et en eau et les lavabos. Leurs occupants peuvent " équiper» l'espace conformément à leurs desiderata aussi souvent qu'ils le souhaitent. En revanche, pour des raisons difficiles à comprendre, des centaines de millions de dollars sont investis dans des écoles "figées dans le temps ». Par exemple, dans la ville de New York, à de rares exceptions près, les nouvelles écoles continuent à être construites 
en étant dotées de cloisons en maçonnerie inamovibles comme si les exigences scolaires des années 80 et 90 figées sur place par I'architecture actuelle allaient demeurer les mêmes pendant tout le XXI e siècle. Or, justement, la plupart de ces écoles sont déjà dépassées le jour de leur inauguration.

Quelle est donc la solution? Celle adoptée par la communauté des affaires n'est pas applicable aux écoles, qui sont moins susceptibles de subir un important réaménagement de leur intérieur tous les 15 ans ou à peu près. En outre, contrairement aux locaux commerciaux et industriels dont les activités sont plus facilement prévisibles, et, de ce fait, pour lesquelles il est plus facile de créer, les écoles exigent une conception beaucoup plus souple. Dans les écoles, des modes d'enseignement divers et des publics de taille différente doivent s'accommoder $d^{\prime}$ un nombre de mètres carrés par occupant dont la norme est une fraction de celle autorisée dans le monde des affaires.

Une façon de concevoir l'école du XXI siècle est de considérer qu'elle est comme un théâtre d'improvisation. Ce théâtre sert à des fins que son concepteur ne pourra jamais toutes imaginer. Un jour donné, ce théâtre pourrait accueillir sur scène une seule personne, un duo, un petit groupe ou même un grand groupe comme une chorale. L'idée est que les occupants définissent l'espace et que les activités réalisées à l'intérieur de cet espace définissent son objet. Elle rompt avec celle des écoles traditionnelles dont les espaces définissent leur objet et dont les occupants doivent vivre dans les limites de cet objet prédéterminé.

\section{Conclusion}

Les conceptions de l'école du XXIe siècle se différencieront sur un point significatif de la scène sans décor qui caractérise le théâtre d'improvisation. Sur une scène sans décor, on s'attend à ce que ce soient les acteurs qui apportent tous les éléments de stimulation. Dans une école, au contraire, la scène doit avoir des éléments avec lesquels les acteurs peuvent interagir, des éléments qui favorisent la diversité des interactions. Par exemple, ses utilisateurs peuvent aménager une aire de repos située autour d'une fontaine et équipée de sièges amovibles de façon très différente de l'aménagement d'une aire similaire mais équipée de sièges fixes. Les salles de cours dotées de cloisons amovibles, de mobilier adaptable et de meubles à cases non fixés devraient favoriser une plus grande diversité de styles d'apprentissage et d'enseignement que celles dont les cloisons sont inamovibles, le mobilier standard et les meubles à cases fixés. La valeur éducative d'une peinture murale sera probablement inférieure à celle d'un mur électronique - qui permettra peut-être aux élèves de s'exprimer artistiquement.

Les espaces ne doivent pas être conçus uniquement pour stimuler l'action. Il est nécessaire que certains espaces encouragent la contemplation. Dans un monde frénétique où des stimuli externes assaillent de tous côtés les individus, les lieux d'apprentissage doivent fournir la possibilité de réfléchir et de ne pas être dérangés, de se ressourcer sur le plan mental et spirituel. Ces espaces doivent être conçus de manière à ce que les apprenants y soient naturellement attirés. Ils doivent être des lieux où le temps s'arrête - du moins l'espace d'un instant.

Pour de plus amples renseignements, contacter :

Prakash Nair, RA, REFP

Président, Urban Educational Facilities for the $21^{\text {st }}$ Century

Directeur, Educational Facilities Planning, Vitetta

53 Haddonfield Road, Suite 306

Cherry Hill, NJ 08002

États-Unis

Télécopie : 18563212009

Prakash@Designshare.com

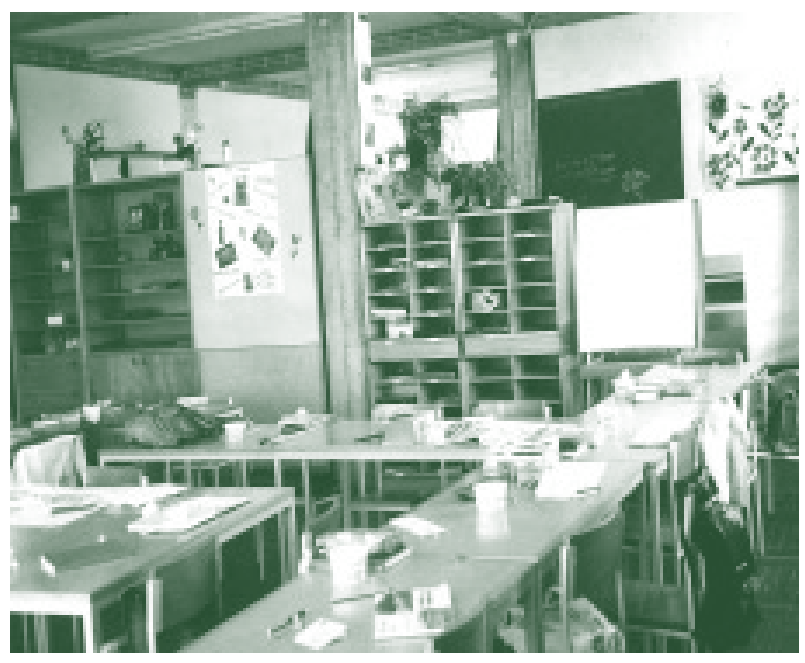

